\title{
Pó de forno de aciaria elétrica na microbiota do solo e no crescimento de soja ${ }^{(1)}$
}

\author{
Rogério Melloni(2), Francisca Alcivânia de Melo Silva( ${ }^{(3)}$, \\ Fátima Maria de Souza Moreira ${ }^{(2)}$ e Antônio Eduardo Furtini Neto(2)
}

\begin{abstract}
Resumo - Devido à grande produção de resíduos de siderurgia, tem sido indicado seu aproveitamento na agricultura como fonte alternativa de micronutrientes. O objetivo deste trabalho foi avaliar o efeito da aplicação do pó de forno de aciaria elétrica na microbiota de solos e sua potencialidade no fornecimento de micronutrientes à soja. O experimento foi realizado em casa de vegetação, com delineamento inteiramente ao acaso, com dois tipos de solo (Latossolo Vermelho-Amarelo (LVA) e Latossolo Vermelho (LV)) e quatro doses de pó de aciaria elétrica $\left(0,0,75,1,50\right.$ e $3,00 \mathrm{~g} \mathrm{vaso}^{-1}$ equivalendo a $0,1,2$ e $\left.4 \mathrm{t} \mathrm{ha}^{-1}\right)$, com quatro repetições. Os solos foram corrigidos e fertilizados antes da adição do resíduo e semeadura de soja inoculada, em vasos de plástico de 1,5 L (duas plantas por vaso). Na floração, coletaram-se as plantas e amostras de solo de todos os tratamentos, para determinação da matéria seca da parte aérea e raízes, teores foliares de $\mathrm{B}, \mathrm{Cu}, \mathrm{Fe}, \mathrm{Mn}, \mathrm{Zn}, \mathrm{Cd}$ e $\mathrm{Pb}$, número de nódulos e sua matéria seca, atividade da nitrogenase dos nódulos, presença de diazotróficos associativos, respiração microbiana, C-biomassa microbiana e $q \mathrm{CO}_{2}$. Há respostas diferenciadas das comunidades microbianas dos solos LVA e LV ao resíduo de siderurgia aplicado, e essas comunidades demonstram maior sensibilidade ao efeito do resíduo de siderurgia do que os parâmetros de crescimento e nodulação da soja. O resíduo de siderurgia apresenta potencial de utilização como fonte de Zn à cultura da soja, nas doses de $0,5 \mathrm{e}$ $1,4 \mathrm{t} \mathrm{ha}^{-1}$ em relação ao LVA e LV, respectivamente.
\end{abstract}

Termos para indexação: resíduo siderúrgico, biomassa, oligoelementos, diazotrofia

\section{Electric aciary oven powder on soil microbiota and growth of soybean}

Abstract - Due to the great production of siderurgical residues, its use has been indicated in agriculture as an alternative source of micronutrients to the plants. The aim of this study was to evaluate the effect of aciary oven powder application on soil microbiota and its potential use as micronutrient source for soybean growth. An experiment was conducted under greenhouse conditions in a completely randomized block design, with Typic Haplorthox (LVA) and Clay Rhodic Hapludox (LV), four levels of siderurgical residue $\left(0 ; 0.75 ; 1.50\right.$ and $3.00 \mathrm{~g} \mathrm{pot}^{-1}$, corresponding to $0,1,2$ and 4 ton ha $\left.{ }^{-1}\right)$, and four replicates. The soils were limed and fertilized before addiction of the residue and sowing of inoculated soybean, in plastic pots of $1.5 \mathrm{~L}$ (two plants per pot). During the blooming of plants, soil samples of all treatments were collected for determination of shoot and root dry matter, shoot concentrations of $\mathrm{B}$, $\mathrm{Cu}, \mathrm{Fe}, \mathrm{Mn}, \mathrm{Zn}, \mathrm{Cd}$ and $\mathrm{Pb}$, number and dry matter of nodules, nitrogenase activity, semi-quantitative evaluation of associative diazotrophics, microbial activity (basal and induced respiration), microbial biomass $\mathrm{C}$, and $q \mathrm{CO}_{2}$. The microbial communities of both soils vary with the siderurgical residue, demonstrating larger sensibility to the effect of the residue than the soybean growth and nodulation parameters. The residue has a potential to be used as source of $\mathrm{Zn}$ to the soybean culture, at doses 0.5 ton $\mathrm{ha}^{-1}$ for LVA and 1.4 ton $\mathrm{ha}^{-1}$ for LV.

Index terms: siderurgical residue, biomass, trace elements, diazotrophy.

(1) Aceito para publicação em 26 de janeiro de 2001.

Parcialmente financiado pela Companhia Siderúrgica BelgoMineira, Belo Horizonte, MG.

(2) Universidade Federal de Lavras (Ufla), Dep. de Ciência do Solo, Caixa Postal 37, CEP 37200-000 Lavras, MG. Bolsista do CNPq. E-mail: rmelloni@hotmail.com, fmoreira@ufla.br, afurtini@ufla.br

(3)Ufla, Dep. de Ciência do Solo. Bolsista da Capes. E-mail: alcivania@hotmail.com

\section{Introdução}

A produtividade de algumas culturas vem sendo limitada pela escassez de micronutrientes, a qual é devida à baixa fertilidade dos solos, remoção desses nutrientes pelas colheitas e utilização de doses elevadas de calcário. $\mathrm{O}$ desenvolvimento acelerado da siderurgia brasileira tem gerado grande quantidade 
de resíduos que podem constituir uma alternativa viável no fornecimento de alguns micronutrientes às plantas e reduzir problemas ambientais ocasionados pelo seu acúmulo. No entanto, uma das limitações para sua utilização é a presença de metais pesados que podem afetar plantas e microrganismos do solo. Estudos anteriores mostraram seu potencial como fonte de micronutrientes para a cultura do sorgo (Nogueira, 1990), milho (Accioly, 1996) e feijoeiro (Silva, 1999), porém não se têm informações sobre seu efeito na microbiota do solo, o qual depende do tipo de solo e da concentração dos metais aplicados (Brookes, 1995). Os metais pesados podem afetar a morfologia, crescimento e metabolismo de microrganismos (Leita et al., 1995), e reduzir, assim, a quantidade e atividade da biomassa microbiana no solo (Bardgett \& Saggar, 1994; Fließbach et al., 1994; Valsecchi et al., 1995).

Os microrganismos do solo estão altamente envolvidos na ciclagem do $\mathrm{N}$ por processos como a fixação de $\mathrm{N}_{2}$, nitrificação, desnitrificação, mineralização e imobilização. Vários estudos com rizóbio comprovaram que concentrações altas de metais pesados podem causar morte, redução da diversidade, diminuição do número e peso de nódulos, e perda de efetividade na fixação do N (Giller et al., 1989; Hirsch et al., 1993). Por outro lado, trabalhos que avaliam resposta de microrganismos diazotróficos endofíticos dos gêneros Herbaspirillum e Azospirillum a metais pesados (Dias-Júnior et al., 1998) são raros, e devem ser valorados por sua importância na funcionalidade dos solos, principalmente nos que são cultivados com gramíneas.

Além de grupos de microrganismos específicos, outras variáveis que podem ser utilizadas para avaliar o impacto da adição de metais pesados ao solo são a respiração, a biomassa microbiana e o quociente metabólico, $q \mathrm{CO}_{2}$, que é a taxa de respiração específica da biomassa (Anderson \& Domsch, 1993), pois são sensíveis a estresses naturais ou antropogênicos, como a adição de compostos tóxicos e metais pesados (Fließbach et al., 1994; Leita et al., 1995; Valsecchi et al., 1995).

O objetivo deste trabalho foi avaliar a utilização do pó de forno de aciaria elétrica como fonte de micronutrientes à soja e o impacto da aplicação na microbiota de solos submetidos a doses desse resíduo.

\section{Material e Métodos}

Amostras do Latossolo Vermelho-Amarelo (LVA) e do Latossolo Vermelho (LV) dos municípios de Itumirim, MG, e São Sebastião da Vitória, MG, respectivamente, foram coletadas $(0-20 \mathrm{~cm})$ e selecionadas pela representatividade da região do Cerrado (Tabela 1). Aplicou-se nas amostras de solo calcário dolomítico $(14 \% \mathrm{MgO}, 35 \% \mathrm{CaO}$ e PRNT $100 \%$ ), para elevar a saturação por bases a $60 \%$ e $200 \mathrm{mg} \mathrm{dm}^{-3}$ de $\mathrm{P}\left(\mathrm{KH}_{2} \mathrm{PO}_{4}\right.$ e $\left._{3} \mathrm{PO}_{4}\right) ; 150 \mathrm{mg} \mathrm{dm}^{-3}$ de K $\left(\mathrm{KH}_{2} \mathrm{PO}_{4}\right)$ e $40 \mathrm{mg} \mathrm{dm}^{-3}$ de $\mathrm{S}\left(\mathrm{CaSO}_{4} \cdot 2 \mathrm{H}_{2} \mathrm{O}\right)$, para $\mathrm{LV}$; e $150 \mathrm{mg} \mathrm{dm}^{-3}$ de $\mathrm{P}, 100 \mathrm{mg} \mathrm{dm}^{-3}$ de $\mathrm{K}$ e $40 \mathrm{mg} \mathrm{dm}^{-3}$ de $\mathrm{S}$ para LVA, utilizando-se as mesmas fontes, antes da adição do resíduo e semeadura de soja (MG/BR46) que recebeu inoculação das estirpes de rizóbio BR96/SEMIA587 e BR29/SEMIA5019. O experimento foi realizado em casa de vegetação, com delineamento inteiramente ao acaso, utilizando-se vasos plásticos de 1,5 L, com duas plantas por vaso, quatro doses do pó de forno de aciaria elétrica $(0,0,75,1,50$ e $3,00 \mathrm{~g} / \mathrm{vaso})$, equivalentes à aplicação de $0,1,2$ e $4 \mathrm{t} \mathrm{ha}^{-1}$, e quatro repetições. A escolha das doses de pó de forno de aciaria elétrica foi baseada no trabalho de Silva (1999) que avaliou doses até $2,9 t$ ha $^{-1}$ desse resíduo na nutrição e produção de feijoeiro cultivado nos mesmos tipos de solo. O resíduo foi fornecido pela Companhia Siderúrgica Belgo-Mineira (Juiz de Fora, MG) cuja composição química se encontra na Tabela 2 . Na floração

Tabela 1. Características químicas e físicas de amostras $(0-20 \mathrm{~cm})$ de Latossolo Vermelho-Amarelo (LVA) e Latossolo Vermelho (LV), antes da aplicação dos tratamentos

\begin{tabular}{lrr}
\hline Características químicas e físicas & LVA & LV \\
\hline $\mathrm{pH} \mathrm{em} \mathrm{água}(1: 2,5)$ & 5,5 & 4,9 \\
$\mathrm{P}\left(\mathrm{mg} \mathrm{dm}^{-3}\right)^{(1)}$ & 1,0 & 1,0 \\
$\mathrm{~K}\left(\mathrm{mg} \mathrm{dm}^{-3}\right)^{(1)}$ & 8,0 & 33,0 \\
$\mathrm{Ca}\left(\mathrm{cmol}_{\mathrm{c}} \mathrm{dm}^{-3}\right)^{(2)}$ & 0,1 & 0,7 \\
$\mathrm{Mg}\left(\mathrm{cmol}_{\mathrm{c}} \mathrm{dm}^{-3}\right)^{(2)}$ & 0,1 & 0,2 \\
$\mathrm{Al}\left(\mathrm{cmol}_{\mathrm{c}} \mathrm{dm}^{-3}\right)^{(2)}$ & 0,0 & 0,3 \\
$\mathrm{Cu}\left(\mathrm{mg} \mathrm{dm}^{-3}\right)^{(3)}$ & 0,9 & 1,8 \\
$\mathrm{Fe}\left(\mathrm{mg} \mathrm{dm}^{-3}\right)^{(3)}$ & 52,4 & 65,9 \\
$\mathrm{Mn}\left(\mathrm{mg} \mathrm{dm}^{-3}\right)^{(3)}$ & 0,7 & 1,6 \\
Zn $\left(\mathrm{mg} \mathrm{dm}^{-3}\right)^{(3)}$ & 0,4 & 0,4 \\
Saturação por bases $(\%)$ & 10,0 & 15,0 \\
Matéria orgânica $\left(\mathrm{g} \mathrm{kg}^{-1}\right)^{(4)}$ & 10 & 33 \\
Argila $\left(\mathrm{g} \mathrm{kg}^{-1}\right)^{(5)}$ & 180 & 730 \\
Silte $\left(\mathrm{g} \mathrm{kg}^{-1}\right)^{(5)}$ & 175 & 130 \\
Areia $\left(\mathrm{g} \mathrm{kg}^{-1}\right)^{(5)}$ & 645 & 140 \\
\hline
\end{tabular}

(1)Extrator Mehlich-1 (Defelipo \& Ribeiro, 1981). (2)Extrator KCl 1 mol L-1 (Defelipo \& Ribeiro, 1981). (3)Extrator DTPA (Embrapa, 1997). (4)Método Walkley \& Black (Embrapa, 1997). ${ }^{(5)}$ Método da pipeta (Embrapa, 1997) 
(63 dias após a semeadura), coletaram-se as plantas e amostras de solo de todos os tratamentos para determinação da matéria seca da parte aérea (MSPA) e das raízes, número e matéria seca de nódulos, atividade de nitrogenase pelo método de redução do acetileno (Kurz \& Larue, 1975), atividade microbiana (respiração basal e induzida, avaliada por cromatografia gasosa, segundo Alef \& Nannipieri, 1995), C-biomassa microbiana (fumigação-extração, conforme Vance et al., 1987), e avaliação semi-quantitativa de diazotróficos endofíticos (Herbaspirillum spp. Azospirillum amazonense, A. lipoferum e A. brasilense), utilizando-se meios de cultura específicos (Magalhães, 1983; Döbereiner et al., 1995) distribuídos em cinco frascos por tratamento. Os elementos $\mathrm{B}, \mathrm{Cu}, \mathrm{Fe}, \mathrm{Mn}, \mathrm{Zn}, \mathrm{Cd}$ e $\mathrm{Pb}$ na parte aérea foram determinados após digestão nitroperclórica e leitura em espectrofotômetro de chama (Malavolta et al., 1997).

Os resultados foram submetidos a análise de variância e regressão através do programa estatístico SANEST (Zonta et al., 1984); na análise dos dados de ocorrência de microrganismos diazotróficos endofíticos e número de nódulos radiculares utilizou-se a transformação raiz quadrada de $(x+0,5)$.

\section{Resultados e Discussão}

\section{Microbiota dos solos}

Não houve efeito da aplicação do resíduo sobre a população de microrganismos diazotróficos endofíticos nos solos estudados, indicando, assim, que a quantidade de metais pesados adicionados não foi suficiente para causar impacto negativo nesses mi-

Tabela 2. Composição química do pó de forno de aciaria elétrica $^{(1)}$.

\begin{tabular}{lc}
\hline Composição química & Teores totais $^{(2)}$ \\
\hline $\mathrm{N}\left(\mathrm{g} \mathrm{kg}^{-1}\right)^{(3)}$ & 0,0 \\
$\mathrm{P}\left(\mathrm{g} \mathrm{kg}^{-1}\right)$ & 1,8 \\
$\mathrm{~K}\left(\mathrm{~g} \mathrm{~kg}^{-1}\right)$ & 26,0 \\
$\mathrm{Ca}\left(\mathrm{g} \mathrm{kg}^{-1}\right)$ & 40,3 \\
$\mathrm{Mg}\left(\mathrm{g} \mathrm{kg}^{-1}\right)$ & 8,2 \\
$\mathrm{Al}\left(\mathrm{g} \mathrm{kg}^{-1}\right)$ & 16,8 \\
$\mathrm{Cu}\left(\mathrm{mg} \mathrm{kg}^{-1}\right)$ & 13.850 \\
$\mathrm{Fe}\left(\mathrm{mg} \mathrm{kg}^{-1}\right)$ & 680.540 \\
$\mathrm{Mn}\left(\mathrm{mg} \mathrm{kg}^{-1}\right)$ & 14.000 \\
$\mathrm{Zn}\left(\mathrm{mg} \mathrm{kg}^{-1}\right)$ & 204.180 \\
$\mathrm{~Pb}\left(\mathrm{mg} \mathrm{kg}^{-1}\right)$ & 26.110 \\
$\mathrm{Cd}\left(\mathrm{mg} \mathrm{kg}^{-1}\right)$ & 118 \\
\hline${ }^{(1)} \mathrm{Accioly} \mathrm{(1996)}$ e Silva (1999). (2) Teores após digestão total com \\
$\mathrm{HF}+\mathrm{HClO}_{4}+\mathrm{HNO}_{3}$ (Mann \& Ritchie, 1993). (3)Método Kjeldahl (Bremner, \\
1965).
\end{tabular}

crorganismos. No entanto, Dias-Júnior et al. (1998) não isolaram esses microrganismos em áreas de deposição de rejeitos da industrialização de $\mathrm{Zn}$, altamente contaminadas por metais pesados (acima de $26 \mathrm{mg} \mathrm{kg}^{-1}$ de $\mathrm{Cd}$, acima de $70 \mathrm{mg} \mathrm{kg}^{-1}$ de $\mathrm{Pb}$ e acima de $1.483 \mathrm{mg} \mathrm{kg}^{-1}$ de $\mathrm{Zn}$ ), o que indica alta sensibilidade aos metais e o potencial de toxicidade do resíduo de siderurgia a essas populações.

Quanto à atividade microbiana dos solos, houve efeito do resíduo na respiração basal no LVA e LV, e respiração induzida no LV (Figura 1). Os maiores valores de respiração basal foram obtidos com dose estimada de 2,4 $\mathrm{t} \mathrm{ha}^{-1} \mathrm{em}$ ambos os solos $(0,38 \mathrm{em}$ LVA e $0,77 \mathrm{mg} \mathrm{kg}^{-1} \mathrm{dia}^{-1}$ de $\mathrm{CO}_{2}$ em LV), mas bem inferiores aos encontrados por Dias-Júnior et al. (1998) em maiores concentrações de metais pesados. Apesar de a atividade microbiana no LV ser aproximadamente o dobro da apresentada no LVA, verificou-se significativa sensibilidade da microbiota do primeiro, em razão das doses aplicadas de resíduo, em face da queda acentuada da respiração induzida com as doses de resíduo. Desta variável, os maiores valores foram encontrados em doses estimadas abaixo de $1 \mathrm{t} \mathrm{ha}{ }^{-1}$ no LV, indicando, assim, o efeito negativo do resíduo na atividade de microrganismos que utilizam preferencialmente fontes de $\mathrm{C}$ prontamente disponíveis, como a glicose, utilizada nessa avaliação. Alguns autores têm verificado que a contaminação do solo por metais pesados não tem efeitos consistentes sobre a respiração microbiana do solo (Bardgett \& Saggar, 1994), enquanto outros têm mostrado um decréscimo pela adição de resíduos orgânicos (Hattori, 1992) ou inorgânicos (Insan et al., 1996), ou um incremento nos valores até um certo nível de contaminação (Leita et al., 1995; Valsecchi et al., 1995). Os resultados obtidos no presente trabalho confirmam a redução da respiração microbiana basal e induzida dos solos que receberam o resíduo, indicando seu efeito negativo, principalmente no LV, que possui uma comunidade microbiana mais ativa que o LVA.

Quanto à biomassa microbiana, houve efeito do resíduo somente quanto ao C-biomassa microbiana do LV (Figura 1). Amostras de LVA que não receberam resíduo apresentaram aproximadamente o dobro da biomassa microbiana que o LV, e não sofreu efeito do resíduo aplicado. No entanto, apesar de a 
biomassa inicial no LV ser menor, o resíduo promoveu aumento da biomassa microbiana até um máximo de $363 \mu \mathrm{g} \mathrm{g}^{-1} \mathrm{de}$, na dose estimada de $2,5 \mathrm{t} \mathrm{ha}^{-1}$, com redução dos valores em doses superiores. Esse resultado indica a existência de comunidades microbianas com respostas diferenciadas ao resíduo, sendo positiva (biomassa e respiração basal) em doses inferiores a 2,5 tha-1 no LV, e sem efeito no LVA. O comportamento diferenciado da biomassa microbiana em solos com variação do teor de matéria orgânica e textura foi relatado por vários autores. Solos contaminados por metais pesados e com maior teor de matéria orgânica tendem a apresentar maiores valores de biomassa, provavelmente em virtude da maior complexação dos metais, reduzindo ou eliminando seu efeito na microbiota (Dias-Júnior et al., 1998). Segundo Lo et al. (1992), os efeitos dos metais pesados dependem da natureza dos complexos formados, com os ácidos húmicos originando
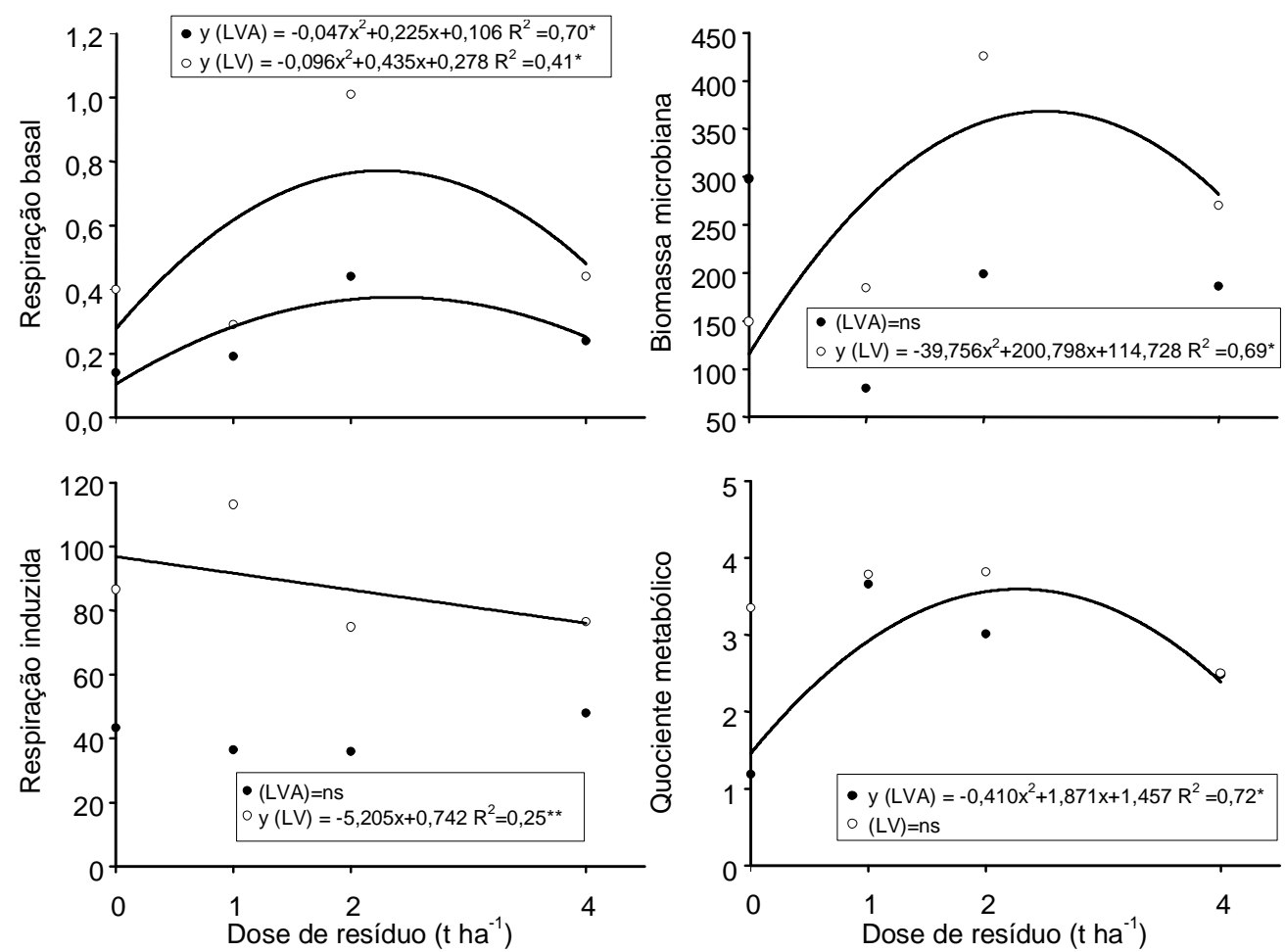

Figura 1. Respiração microbiana basal $\left(\mathrm{mg} \mathrm{CO} \mathrm{kg}^{-1}\right.$ de solo dia $\left.{ }^{-1}\right)$, respiração microbiana induzida ( $m g \mathrm{CO}_{2} \mathrm{~kg}^{-1}$ de solo dia ${ }^{-1}$ ), biomassa microbiana ( $\mu \mathrm{g} \mathrm{C} \mathrm{g}{ }^{-1}$ de solo) e quociente metabólico ( $\mu \mathrm{g} \mathrm{C}-\mathrm{CO}_{2} 10^{4} \mathrm{~h}^{-1} / \mu \mathrm{g}$ C-biomassa g ${ }^{-1}$ de solo) no Latossolo Vermelho-Amarelo (LVA) e Latossolo Vermelho (LV), em razão das doses do resíduo. complexos de baixa solubilidade e os ácidos orgânicos aumentando sua mobilidade e disponibilidade biológica. No presente trabalho, o LV apresentou maiores teores de matéria orgânica e argila (Tabela 1), os quais possivelmente contribuíram para reduzir a disponibilidade dos metais pesados aplicados, permitindo um aumento da biomassa microbiana até a dose estimada de $2,5 \mathrm{t} \mathrm{ha}^{-1}$.

A partir dos resultados da respiração basal e C-biomassa microbiana, calculou-se o quociente metabólico $\left(q \mathrm{CO}_{2}\right)$, que representa a quantidade de $\mathrm{C}-\mathrm{CO}_{2}$ liberada por unidade de C-microbiano. Maiores valores são encontrados em condições mais adversas à população microbiana, onde se gasta mais C oxidável para sua manutenção. Isto ocorre geralmente em solos ácidos, naqueles que receberam adição recente de substratos orgânicos e nos impactados por metais pesados (Anderson \& Domsch, 1993; Fließbach et al., 1994; Grisi, 1995; Valsecchi et al., 
1995; Dias-Júnior et al., 1998). No presente trabalho, verificou-se efeito de resíduo somente no $q \mathrm{CO}_{2}$ do LVA, com maior valor $\left(3,6 \mu \mathrm{g} \mathrm{C}-\mathrm{CO}_{2} \cdot 10^{4} \mathrm{~h}^{-1} / \mu \mathrm{g}\right.$ C-biomassa $\mathrm{g}^{-1}$ de solo seco) na dose estimada de 2,3 $\mathrm{t} \mathrm{ha}^{-1}$, indicando o máximo de estresse aos metais pesados aplicados nessa dose (Figura 1). Essa variável indicou que para o LV, a microbiota não sofreu efeito da adição do resíduo, provavelmente por causa da presença de maiores teores de argila e matéria orgânica, os quais reduzem a disponibilidade dos metais pesados aplicados (Alloway, 1990; Costa, 1991).

\section{Nodulação, crescimento e teores foliares de $\mathrm{B}, \mathrm{Cu}$, Fe, Mn, Zn, Cd e Pb em soja}

Verificou-se efeito do resíduo no número de nódulos de plantas cultivadas em ambos os tipos de solo, na matéria seca de nódulos de plantas no LVA e na atividade de nitrogenase nos nódulos de plantas no LV (Figura 2). Plantas cultivadas em LV apresentaram maior número, matéria seca e atividade de nódulos do que as cultivadas em LVA, em todas as doses de resíduo. No entanto, no número de nódulos de soja cultivada em ambos os solos verificou-se que não houve alteração dos valores na dose 0 e $4 \mathrm{t} \mathrm{ha}^{-1}$; isto indica que o resíduo não afetou negativamente a nodulação no presente estudo. Contrariamente, Reddy et al. (1983) verificaram efeito negativo de metais pesados na sobrevivência de Bradyrhizobium japonicum em solo tratado com lodo de esgoto, o qual reduz o potencial de nodulação em soja. O impacto dos metais pesados na nodulação da soja do presente trabalho foi reduzido no solo LV, possivelmente em virtude dos maiores teores de argila e matéria orgânica e melhor fertilidade desse solo, em relação ao LVA. Quanto à atividade da nitrogenase, verificou-se aumento considerável na última dose do resíduo (Figura 2). Possível hipótese desse comportamento seria a substituição do molibdênio da molécula da nitrogenase por outro elemento presente em quantidade significativa no resíduo, já que sua substituição foi comprovada em vários estudos (Pimentel, 1991; Bishop \& Premakumar, 1992).

Quanto ao crescimento da soja até o florescimento, verificou-se que houve efeito do resíduo na matéria seca da parte aérea (MSPA) das plantas cultivadas
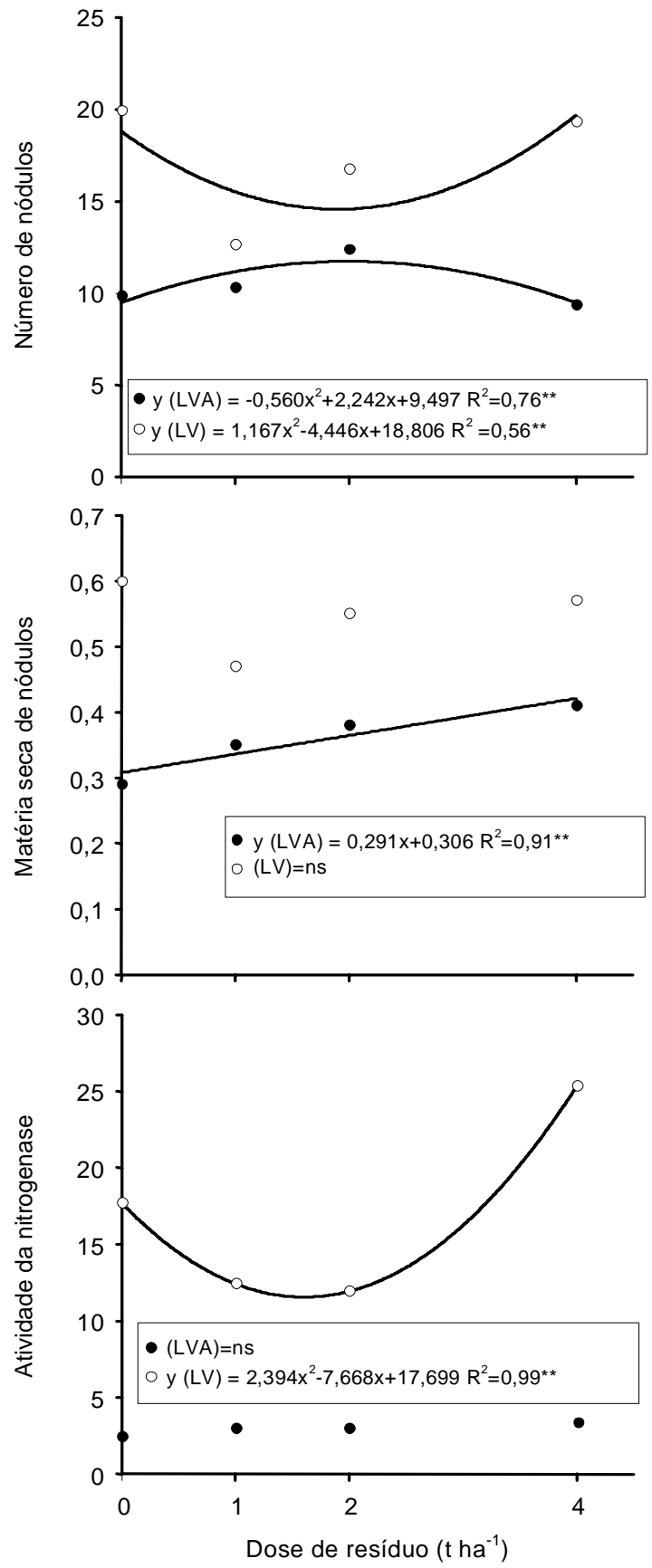

Figura 2. Número de nódulos por vaso (dados transformados em raiz quadrada de $\mathrm{x}+0,5)$, matéria seca de nódulos $\left(\mathrm{g} \mathrm{vaso}^{-1}\right)$ e atividade da nitrogenase $\left(\mu \mathrm{mol} \mathrm{C}_{2} \mathrm{H}_{4}\right.$ reduzido $\mathrm{h}^{-1}$ ) em nódulos de soja cultivada em Latossolo Vermelho-Amarelo (LVA) e Latossolo Vermelho (LV), em razão das doses do resíduo. 
em ambos os solos e na matéria seca de raízes das plantas no LVA (Figura 3). Plantas cultivadas no solo LV apresentaram os maiores valores dessas variáveis, em todas as doses do resíduo, com hipóteses ligadas à menor disponibilidade de metais e maior fertilidade desse solo, em relação ao LVA. Assim, verificouse que não houve efeito negativo da aplicação do resíduo no crescimento da soja cultivada nos dois tipos de solo, contrariamente ao observado no caso da atividade e biomassa microbianas e $q \mathrm{CO}_{2}$ (Figura 1). Com esses resultados, pode-se indicar que parâmetros microbiológicos apresentam maior sensibilidade aos efeitos da atividade antropogênica no solo, o que concorda com diversos autores (Doran et al., 1994; Brookes, 1995; Kennedy \& Papendick, 1995; Bruggen \& Semenov, 2000; Doran \& Zeiss, 2000).

No LVA, houve efeito do resíduo nos teores foliares de $\mathrm{B}, \mathrm{Cu}, \mathrm{Mn}, \mathrm{Zn}, \mathrm{Pb}$ e $\mathrm{Cd}$, enquanto no $\mathrm{LV}$ houve efeito somente quanto aos teores foliares de $\mathrm{Cu}, \mathrm{Mn}$ e $\mathrm{Zn}$ (Tabela 3). As faixas adequadas dos teores foliares dos micronutrientes na cultura da soja são 21 a $55 \mathrm{mg} \mathrm{kg}^{-1} \mathrm{de} \mathrm{B}, 10$ a $30 \mathrm{mg} \mathrm{kg}^{-1} \mathrm{de} \mathrm{Cu}, 51$ a $350 \mathrm{mg} \mathrm{kg}^{-1} \mathrm{de} \mathrm{Fe}, 21$ a $100 \mathrm{mg} \mathrm{kg}^{-1}$ de Mn e 21 a $50 \mathrm{mg} \mathrm{kg}^{-1}$ de Zn (Malavolta et al., 1997).

Considerando que os maiores valores de MSPAe matéria seca de raízes ocorreram na maior dose de resíduo aplicada $\left(4 \mathrm{tha}^{-1}\right)$, em ambos os solos, os teores foliares de $\mathrm{B}, \mathrm{Cu}, \mathrm{Fe}$ e $\mathrm{Mn}$ nesta dose foram adequados, enquanto os teores foliares de $\mathrm{Zn}$ foram de, aproximadamente, $139 \mathrm{mg} \mathrm{kg}^{-1}$ nas plantas cultivadas no LVA, e de $93 \mathrm{mg} \mathrm{kg}^{-1}$ nas cultivadas no LV. Com isso, a dose de resíduo de $4 \mathrm{tha}^{-1}$ promoveria teores foliares de Zn inadequados à soja, limitando a indicação do pó de forno de aciaria elétrica para essa cultura, nesses solos. Para se alcançar o teor foliar máximo desse micronutriente ( $\left.50 \mathrm{mg} \mathrm{kg}^{-1}\right)$, a quan-
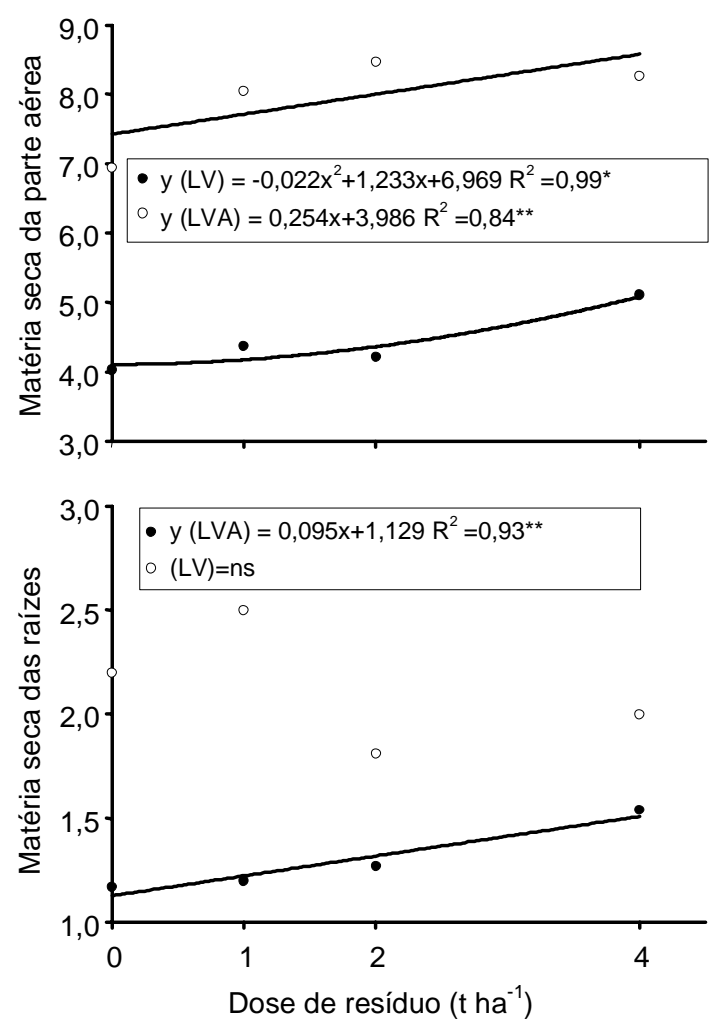

Figura 3. Matéria seca $\left(\mathrm{g} \mathrm{vaso}^{-1}\right)$ da parte aérea e das raízes de soja cultivada em Latossolo Vermelho-Amarelo (LVA) e Latossolo Vermelho (LV), em razão das doses de resíduo.

Tabela 3. Equações de regressão dos teores foliares de $\mathrm{B}, \mathrm{Cu}, \mathrm{Fe}, \mathrm{Mn}, \mathrm{Zn}, \mathrm{Pb}$ e Cd em plantas de soja cultivadas em amostras de Latossolo Vermelho-Amarelo (LVA) e Latossolo Vermelho (LV) contendo doses de resíduo de siderurgia.

\begin{tabular}{lllllll}
\hline Elemento & \multicolumn{2}{c}{ LVA } & & \multicolumn{2}{c}{$\mathrm{LV}$} \\
\cline { 2 - 3 } & Equação & $\mathrm{R}^{2}$ & & Equação & $\mathrm{R}^{2}$ \\
$\mathrm{~B}$ & $\mathrm{y}=-2,36 \mathrm{x}^{2}+14,47 \mathrm{x}+28,60$ & $0,96^{* *}$ & & $\mathrm{~ns}$ & - \\
$\mathrm{Cu}$ & $\mathrm{y}=-0,27 \mathrm{x}+6,34$ & $0,42^{*}$ & & $\mathrm{y}=0,68 \mathrm{x}+8,25$ & $0,92^{* *}$ \\
$\mathrm{Mn}$ & $\mathrm{ns}$ & - & & $\mathrm{ns}$ & - \\
$\mathrm{Zn}$ & $\mathrm{y}=4,35 \mathrm{x}+26,14$ & $0,97^{* *}$ & & $\mathrm{y}=4,70 \mathrm{x}^{2}-26,13 \mathrm{x}+50,58$ & $0,97^{* *}$ \\
$\mathrm{~Pb}$ & $\mathrm{y}=-8,50 \mathrm{x}^{2}+63,88 \mathrm{x}+19,36$ & $0,99^{* *}$ & $\mathrm{y}=17,62 \mathrm{x}+25,90$ & $0,98^{* *}$ \\
$\mathrm{Cd}$ & $\mathrm{y}=-4,03 \mathrm{x}^{2}+23,07 \mathrm{x}+6,42$ & $0,70^{* *}$ & $\mathrm{~ns}$ & - \\
\hline
\end{tabular}

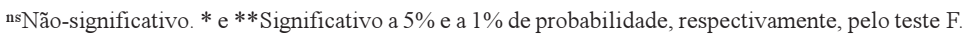


tidade de resíduo a ser indicada seria de $0,5 \mathrm{tha}^{-1} \mathrm{no}$ LVA e $1,4 \mathrm{t} \mathrm{ha}^{-1}$ no LV.

Kabata-Pendias \& Pendias (1984) consideram normais ou suficientes valores de 0,05 a $0,2 \mathrm{mg} \mathrm{kg}^{-1}$ de $\mathrm{Cd}$ e de 5 a $10 \mathrm{mg} \mathrm{kg}^{-1}$ de $\mathrm{Pb}$, e excessivos ou tóxicos, teores foliares de 5 a 30 e de 30 a $300 \mathrm{mg} \mathrm{kg}^{-1}$, respectivamente. Assim, na quantidade indicada de resíduo suficiente para atingir o teor máximo de $\mathrm{Zn}$ foliar $\left(0,5 \mathrm{t} \mathrm{ha}^{-1}\right.$ no LVA e $1,4 \mathrm{t} \mathrm{ha}{ }^{-1}$ no $\left.\mathrm{LV}\right)$, os teores foliares estimados de $\mathrm{Cd}$ e $\mathrm{Pb}$ seriam em torno de $0 \mathrm{e}$ $12 \mathrm{mg} \mathrm{kg}^{-1}$ de $\mathrm{Cd} \mathrm{e} \mathrm{Pb}$, nas plantas cultivadas em ambos os solos, e, portanto, dentro da faixa de teores considerados normais à cultura.

\section{Conclusões}

1. Há respostas diferenciadas das comunidades microbianas dos solos LVA e LV ao resíduo de siderurgia aplicado.

2. A atividade, biomassa e $q \mathrm{CO}_{2}$ são mais sensíveis ao efeito do resíduo de siderurgia do que a produção de matéria seca da parte aérea e radicular, número e matéria seca de nódulos e atividade da nitrogenase da soja.

3. A atividade microbiana dos solos LVA e LV é reduzida em doses superiores a 2,4 t ha- ${ }^{-1}$ (respiração basal) e superiores a $1 \mathrm{t} \mathrm{ha}^{-1}$ no $\mathrm{LV}$ (respiração induzida).

4. A biomassa microbiana no LVA não varia com o resíduo, enquanto no $L V$ ocorre aumento até a dose $2,5 \mathrm{tha}^{-1}$, comportamento inverso ao apresentado pelo $q \mathrm{CO}_{2}$.

5. As doses do resíduo não afetam as populações de microrganismos diazotróficos endofíticos em ambos os solos.

6 . As doses $0,5 \mathrm{tha}^{-1}(\mathrm{LVA})$ e $1,4 \mathrm{t} \mathrm{ha}^{-1}(\mathrm{LV})$ estimadas do resíduo de siderurgia para o fornecimento de quantidades adequadas de Zn para soja não são suficientes para causarem toxidez de $\mathrm{Cd}$ e $\mathrm{Pb}$ nessa leguminosa.

\section{Referências}

ACCIOLY,A. M. A. Pó de forno de siderurgia como fonte de micronutrientes e seu efeito no solo e na planta Lavras: Ufla, 1996. 70 p. Dissertação de Mestrado.

ALEF, K.; NANNIPIERI, P. (Ed.). Methods in applied soil microbiology and biochemistry. London: Academic, 1995. $576 \mathrm{p}$
ALLOWAY, B. J. Cadmium. In: ALLOWAY, B. J. Heavy metals in soils. Glasgow: Academic \& Professional, 1990. p. $100-124$

ANDERSON, T. H.; DOMSCH, K. H. The metabolic quotient for $\mathrm{CO}_{2}\left(q \mathrm{CO}_{2}\right)$ as a specific activity parameter to assess the effects of environmental conditions, such as $\mathrm{pH}$, on the microbial biomass of forest soils. Soil Biology and Biochemistry, Oxford, v. 25, n. 3, p. 393-395, Mar. 1993.

BARDGETT, R. D.; SAGGAR, S. Effects of heavy metal contamination on the short-term decomposition of labeled ${ }^{14} \mathrm{C}$ glucose in a pasture soil. Soil Biology \& Biochemistry, Oxford, v. 26, n. 6, p. 727-733, June 1994

BISHOP, P. E.; PREMAKUMAR, R. Alternative nitrogen fixation systems. In: STACEY, G.; BURRIS, R. H.; EVANS, H. J. (Ed.). Biological nitrogen fixation. New York: Chapman \& Hall, 1992. p. 736-762.

BREMNER, J. M. Total nitrogen. In: BLACK, C. A. (Ed.). Methods of soil analysis. Madison: American Society of Agronomy, 1965. part 2, p. 1149-1178

BROOKES, P. C. The use of microbial parameters in soil pollution by heavy metals. Biology and Fertility of Soils, Berlin, v. 19, n. 4, p. 269-279, Mar. 1995

BRUGGEN, A. H. C. van; SEMENOV, A. M. In search of biological indicators for soil health and disease suppression. Applied Soil Ecology, Amsterdam, v. 15, p. $13-24,2000$

COSTA, E. D. Adsorção e competição de alguns metais por ácidos húmicos extraídos de um Latossolo Húmico da região de Araponga, Minas Gerais. Viçosa, MG: UFV, 1991. 71 p. Dissertação de Mestrado.

DEFELIPO, B. V.; RIBEIRO, A. C. Análise química do solo: metodologia. Viçosa, MG: UFV, 1981. 17 p. (Boletim, 29).

DIAS-JÚNIOR, H. E.; MOREIRA, F. M. S.; SIQUEIRA, J. O.; SILVA, R. Metais pesados, densidade e atividade microbiana em solo contaminado por rejeitos de indústria de zinco. Revista Brasileira de Ciência do Solo, Viçosa, v. 22, n. 4, p. 631-640, out./dez. 1998.

DÖBEREINER, J.; BALDANI, V. L. D.; BALDANI, J. I. Como isolar e identificar bactérias diazotróficas de plantas não-leguminosas. Brasília: Embrapa-SPI, 1995. 60 p.

DORAN, J. W.; ZEISS, M. R. Soil health and sustainability: managing the biotic component of soil quality. Applied Soil Ecology, Amsterdam, v. 15, p. 3-11, 2000

Pesq. agropec. bras., Brasília, v. 36, n. 12, p. 1547-1554, dez. 2001 
DORAN, J. W.; COLEMAN, D. C.; BEZDICEK, D. F.; STEWART, B. A. (Ed.). Defining soil quality for a sustainable environment. Madison: American Society of Agronomy, 1994. 244 p.

EMBRAPA. Centro Nacional de Pesquisa de Solos (Rio de Janeiro, RJ). Manual de métodos de análise de solos 2. ed. Rio de Janeiro, 1997. 212 p.

FLIEßBACH, A.; MARTENS, R.; REBER, H. H. Soil microbial biomass and microbial activity in soils treated with heavy metal contaminated sewage sludge. Soil Biology \& Biochemistry, Oxford, v. 26, n. 9, p. 12011205, Sept. 1994.

GLLER, K. E.; McGRATH, S. P.; HIRSCH, P. R. Absence of nitrogen-fixation in clover grown on soil subject to long term contamination with heavy metals is due to survival of only ineffective Rhizobium. Soil Biology \& Biochemistry, Oxford, v. 21, n. 6, p. 841-848, June 1989

GRISI, B. M. Biomassa e atividade de microrganismos do solo: revisão metodológica. Revista Nordestina de Biologia, João Pessoa, v. 10, n. 1, p. 1-22, 1995.

HATTORI, H. Influence of heavy metals on soil microbial activities. Soil Science and Plant Nutrition, Tokyo, v. 38, n. 1, p. 93-100, 1992.

HIRSCH, P. R.; JONES, M. J.; McGRATH, S. P.; GLLER, K. E. Heavy metals from past applications of sewage sludge decrease the genetic diversity of Rhizobium leguminosarum biovar trifolii populations. Soil Biology \& Biochemistry, Oxford, v. 25, n. 11, p. 1485-1490, Nov. 1993

INSAN, H.; HUTCHINSON, T. C.; REBER, H. H. Effects of heavy metal stress on the metabolic quotient of the soil microflora. Soil Biology \& Biochemistry, Oxford, v. 28, n. 4/5, p. 691-694, 1996.

KABATA-PENDIAS, A.; PENDIAS, H. Trace elements in soil and plants. Boca Raton: CRC, 1984. 315 p.

KENNEDY, A. C.; PAPENDICK, R. I. Microbial characteristics of soil quality. Journal of Soil and Water Conservation, Ankeny, v. 50, p. 243-248, 1995.

KURZ, W. G. W.; LARUE, T. A. Nitrogenase activity in rhizobia in absence of plant host. Nature, London, v. 256, n. 5516, p. 407-408, July 1975

LEITA, L.; NOBILE, M. de; MUHLBACHOVA, G.; MONDONI, C.; MARCHIOL, L.; ZERBI, G.
Bioavailability and effects of heavy metals on soil microbial biomass survival during laboratory incubation. Biology and Fertility of Soils, Berlin, v. 19, n. 2/3, p. 103-108, Feb. 1995

LO, K. L. S.; YANG, W. F.; LIN, Y. C. Effects of organic matter on the specific adsorption of heavy metals by soils. Toxicological and Environmental Chemistry, Chur, v. 34, p. 139-153, 1992.

MAGALHÃES, F. M. M. Caracterização e distribuição de uma nova espécie de bactéria fixadora de nitrogênio. Manaus: Universidade do Amazonas, 1983. 89 p. Dissertação de Mestrado.

MALAVOLTA, E.; VITTI, G. C.; OLIVEIRA, S. A. Avaliação do estado nutricional das plantas: princípios e aplicações. Piracicaba: Associação Brasileira para Pesquisa da Potassa e do Fosfato, 1997. 210 p.

MANN, S. S.; RITCHIE, G. S. P. The influence of $\mathrm{pH}$ on the forms of cadmium in far West Australian soils. Australian Journal of Soil Research, Collingwood, v. 31, p. $255-270,1993$.

NOGUEIRA, A. V. Eficiência agronômica como fertilizante de um lodo de esgoto e de dois resíduos provenientes de indústria siderúrgica. Viçosa, MG: UFV, 1990. 85 p. Dissertação de Mestrado.

PIMENTEL, F. P. A nitrogenase (V- $\mathbf{N}_{2}$-ase) em Azospirillum. Belo Horizonte: UFMG, 1991. 80 p. Dissertação de Mestrado.

REDDY, G. B.; CHENG, C. N.; DUNN, S. J. Survival of Rhizobium japonicum in soil-sludge environment. Soil Biology \& Biochemistry, Oxford, v. 15, n. 3, p. 343-345, May/June 1983

SILVA, F. A. de M. Fracionamento e biodisponibilidade de metais para o feijoeiro (Phaseolus vulgaris L.) em solos tratados com pó de forno de aciaria elétrica. Lavras: Ufla, 1999. 75 p. Dissertação de Mestrado.

VALSECCHI, G.; GIGLIOTTI, C.; FARINI,A. Microbial biomass, activity, and organic matter accumulation in soils contaminated with heavy metals. Biology and Fertility of Soils, Berlin, v. 20, n. 4, p. 253-259, Sept. 1995.

VANCE, E. D.; BROOKES, P. C.; JENKINSON, D. S. An extraction method for measuring microbial biomass C. Soil Biology \& Biochemistry, Oxford, v. 19, n. 6, p. 703-707, June 1987.

ZONTA, E. P.; MACHADO, A. A.; SILVEIRA JÚNIOR, $P$. Sistemas de análise estatística para microcomputadores (SANEST). Pelotas: Ufpel, 1984. 151 p. 